\title{
Mechanism of Astragalus polysaccharides in attenuating insulin resistance in Rats with type 2 diabetes mellitus via the regulation of liver microRNA-203a-3p
}

\author{
ZITAI WEI $^{1}$, SIYING WENG ${ }^{2}$, LEI WANG ${ }^{3}$ and ZHUJUN MAO ${ }^{3}$ \\ ${ }^{1}$ Department of Chemistry of Traditional Chinese Medicine, Medical College, Quzhou College of Technology, \\ Quzhou, Zhejiang 324000; ${ }^{2}$ Department of Endocrinology, Ningbo Municipal Hospital of TCM, Affiliated Hospital of \\ Zhejiang Chineses Medical University, Ningbo, Zhejiang 315000; ${ }^{3}$ Department of Clinical Foundation of \\ Chinese Medicine, College of Basic Medicine, Zhejiang Chinese Medical University, Hangzhou, Zhejiang 310053, P.R. China
}

Received August 10, 2017; Accepted November 9, 2017

DOI: $10.3892 / \mathrm{mmr} .2017 .8084$

\begin{abstract}
Insulin resistance (IR) is a common feature of type 2 diabetes mellitus (T2DM). Astragalus polysaccharides (APS) is a natural medicine that is used to treat T2DM. However, the mechanism by which APS regulates micro (mi)RNA in the treatment of IR has not been investigated. The purpose of the present study was to investigate differential miRNA expression between normal, T2DM model and APS treatment rats, as well as changes in miRNA and its downstream gene expression levels after APS treatment in T2DM Goto Kakizaki (GK) rats. Results suggested that miRNA (miR)-203a-3p expression level was significantly decreased in the liver of T2DM GK rats. Furthermore, it was identified that glucose-regulated protein (GRP)78 was the target gene of miR-203a-3p. GRP78 mRNA and protein expression levels of GRP78, CAAT-enhancer-binding protein homologous protein (CHOP), phosphorylated-c-Jun N-terminal kinase (pJNK)1,
\end{abstract}

Correspondence to: Dr Zhujun Mao, Department of Clinical Foundation of Chinese Medicine, College of Basic Medicine, Zhejiang Chinese Medical University, 548 Binwen Road, Hangzhou, Zhejiang 310053, P.R. China

E-mail:maozhujun0107@163.com

Abbreviations: APS, Astragalus polysaccharides; HFD, high-fat diet; miRNA, microRNA; GK, Goto Kakizaki; GRP78, glucose-regulated protein 78; BW, body weight; PBG, plasma blood glucose; HOMA-IR, homeostasis model assessment index for insulin resistance; TC, total cholesterol; TG, triglycerides; LDL-C, low-density lipoprotein-cholesterol; HDL-C, high-density lipoprotein-cholesterol; TNF- $\alpha$, tumor necrosis factor- $\alpha$; RT-qPCR, reverse transcription-quantitative polymerase chain reaction; GADD153/CHOP, growth arrest and DNA-damage inducible transcript 3/C/EBP homologous protein; JNK1, c-Jun N-terminal kinase 1; pJNK1, phosphorylation of c-Jun N-terminal kinase 1

Key words: Astragalus polysaccharides, endoplasmic reticulum stress, GRP78, insulin resistance, microRNA-203a and caspase-12 were significantly increased in the liver of T2DM GK rats. Furthermore, miR-203a-3p was upregulated following APS treatment, and the protein expression levels of GRP78, CHOP, pJNK1 and caspase-12 were significantly decreased. In addition, miR-203a-3p overexpression in IR cells decreased the protein expression levels of these factors and anti-miR-203a-3p produced the opposite result. These findings provided evidence that miR-203a-3p may have a functional role in endoplasmic reticulum stress (ERS) signaling in the liver of T2DM GK rats. In addition, APS attenuated IR in T2DM, likely through upregulating or maintaining the miR-203a-3p expression levels, decreasing GRP78 mRNA and protein expression levels and regulating the protein expression of the ERS signaling pathway.

\section{Introduction}

Insulin resistance (IR) is an important part of the pathogenesis of type 2 diabetes mellitus (T2DM). MicroRNA affects the secretion of insulin and protein expression of insulin target organs as a regulatory factor before protein transcription (1). Several studies have reported changes in MicroRNAs (miRNAs) in human or animal DM models (2), and recommended plasma miRNAs as the underlying biomarker of T2DM (3).

Astragalus polysaccharides (APS, the main bioactive ingredient of Astragalus) is a macromolecular polysaccharide extracted from the dry root of traditional Chinese medicine Astragalus, which has a good therapeutic effect on diabetes (4). Most previous studies on the action mechanism of APS for DM focused on insulin sensitivity, insulin secretion, glucolipid metabolism in the liver, reduced intestinal absorption of glucose, and modulation of gut microbiota, antioxidant and anti-Inflammatory activities (5-7). However, few studies have reported the mechanism of APS at RNA transcriptional level. Whether the role of APS in improving IR is associated with the regulation of liver microRNAs and its underlying mechanism have not been reported. This study used Goto-Kakizaki (GK) rats to establish a T2DM rat model, The aim of the present study was to see whether APS could induce miRNA change 
in the liver of diabetic rats, and how this change affects the downstream genes in Goto Kakizaki (GK) rats, APS-treated rats and Wistar rats.

\section{Materials and methods}

Experimental animals. Breeds and strains: A total of 20 specific-pathogen-free (SPF) male GK rats (aged 10 weeks) and 10 SPF male Wistar rats (aged 4 weeks) were purchased from the Shanghai Experimental Animal Center of Chinese Academy of Sciences/Shanghai Laboratory Animal Center [Production license: SCXK (Hu) 2007-0005].

SPF-level barrier system rats were fed in the laboratory at $23 \pm 1^{\circ} \mathrm{C}$ and humidity $50-70 \%$. Moreover, the light was 150-200 lx, and bright and dark phases were alternated every $12 \mathrm{~h}$ (6:00 a.m. to 6:00 p.m.). The noise was less than $50 \mathrm{~dB}$, and the use license was SYXK (Zhejiang) 2008-0115.

Drinking water: Running water was filtered, sterilized, and placed in an autoclaved drinking water bottle. The rats had free access to drinking water. Feedstuff: The rats were fed pellets irradiated with Co60 for sterilization. Feeding style: The rats had free access to food and drinks, and plenty of feedstuff and water were kept in the cages. Each cage contained two to three rats. Each rat was weighed and marked with a number before starting the experiments.

The animal experiment procedures were conducted according to guidelines of the National Institutes of Health (NIH), and approved by the Ethics Committee of the Experimental Animal Centre of Zhejiang Chinese Medical University (Approval no. SYXK (ZHE) 2008-0115).

Reagents and instruments. The reagents included APS (Sigma-Aldrich; Merck KGaA, Darmstadt, Germany), TRIzol (Invitrogen; Thermo Fisher Scientific, Inc., Waltham, MA, USA), blood glucose reagent strips (Roche Diagnostics, Basel, Switzerland), insulin enzyme-linked immunosorbent assay (ELISA) kit (R\&D Systems, Inc., Minneapolis, MN, USA), urethane (CAS no. 51-79-6; Shanghai Shanpu Co., Ltd., Shanghai, China), reverse transcription kit (Thermo Fisher Scientific, Inc.), miScript II reverse transcription (RT) kit (Qiagen GmbH, Hilden, Germany), miRcute miRNA quantitative polymerase chain reaction (qPCR) detection kit (SYBR-Green) (Tiangen Biotech Co., Ltd., Beijing, China), whole-protein extraction kit, bicinchoninic acid (BCA) protein quantification kit (KGI Biotechnology Development Co., Ltd., Nanjing, China), polyvinylidene difluoride (PVDF) membrane (EMD Millipore, Billerica, MA, USA), anti-glucose-regulated protein (GRP)78 antibody, anti-DNA-damage inducible transcript 3 (GADD153/CHOP) antibody, anti-c-Jun N-terminal kinase 1 (JNK1) antibody, anti-phosphorylation-c-Jun N-terminal kinase 1 (pJNK1) antibody, anti-caspase-12 antibody, anti-glyceraldehyde 3-phosphate dehydrogenase (GAPDH) antibody (Abcam, Cambridge, UK), bovine serum albumin, sodium dodecyl sulfate-polyacrylamide gel electrophoresis (SDS-PAGE) gel kit, horseradish peroxidase (HRP)-labeled goat anti-mouse immunoglobulin G ( $\operatorname{IgG})$, HRP-labeled goat anti-rabbit IgG (Beijing Zhongshan Jinqiao Biological Technology Co., Ltd., Beijing, China), prestained marker (Thermo Fisher Scientific, Inc.), and enhanced chemiluminescence (ECL) developer (Thermo Fisher Scientific,
Inc.). Moreover, other reagents were domestic analytical reagents. The instruments included Accu-Chek Advantage blood glucose meter (Roche Diagnostics GmbH, Mannheim, Germany), AG204-electronic analytical balance (MettlerToledo. Instruments Co., Ltd., Shanghai, China), VarioScan Flash Multi-functional Microplate Reader (Thermo Fisher Scientific, Inc.), low-temperature centrifuge (Eppendorf, Hamburg, Germany), quantitative PCR instrument (Applied Biosystems; Thermo Fisher Scientific, Inc.), and gel imaging system (Bio-Rad Laboratories, Inc., Hercules, CA, USA).

Animal screening and grouping administration. $20 \mathrm{SPF}$ male GK rats (aged 10 weeks) and 10 Wistar male rats (aged 4 weeks) were selected and fed for 6 week before starting the experiments. The animals had free access to tap water. GK rats were fed high-fat diet containing $10 \%$ fat, whereas Wistar rats were fed ordinary feed. The rats were fasted food, but not water, every other night for $12 \mathrm{~h}$. The weight was measured, and the tail vein blood was extracted. Twenty GK rats were randomly assigned into two groups: Model and APS groups with 10 rats in each group. Ten normal Wistar rats were selected as the control group. The APS group was given APS $(500 \mathrm{mg} / \mathrm{kg} / \mathrm{day})$ intragastrically, whereas the rats in the model and control groups were given the same volume of normal saline, once daily for 8 weeks.

Area under the curve of blood glucose. During the experimental period, the body weights were measured at weekly intervals, and all animals received Random plasma glucose tests before administered and at the 2nd, 4th, 6th and 8th w after treatment. Blood samples were obtained from tail vessels, blood glucose values were determined by glucose oxidase method (Toecho Super II, Kagawa, Japan).

Determination of fasting serum insulin and calculation of HOMA-IR index. Fasting serum insulin was determined using ELISA kit for insulin following the manufacturer's protocol. Using an ELISA reader, the absorbance of the reaction product was read at $450 \mathrm{~nm}$. IR was assessed using the homeostasis model assessment index for IR (HOMA-IR index) using the formula reported by Matthews et al (8): HOMA-IR index=[fasting glucose $(\mathrm{mmol} / \mathrm{l}) \mathrm{x}$ fasting insulin $(\mu \mathrm{U} / \mathrm{ml})] / 22.5)$.

Measurement of blood lipids. After the last administration, the rats were fasted, but with free access to water, for $12 \mathrm{~h}$. They were anesthetized by injecting $20 \%$ urethane $(1.2 \mathrm{~g} / \mathrm{kg})$ intraperitoneally, and $5 \mathrm{ml}$ of blood was extracted from the abdominal aorta. All rats executed by the way of cervical spine dislocation. Of this, $1 \mathrm{ml}$ was placed in a centrifuge tube without anticoagulant, stored at $4^{\circ} \mathrm{C}$ for $10 \mathrm{~min}$, and centrifuged at $3,000 \mathrm{x}$ g for $15 \mathrm{~min}$. The serum was separated, and the levels of TC, TG, HDL-c, and LDL-c were measured using an automatic biochemical analyzer.

Measurement of the TNF- $\alpha$ level in the serum of rats using the ELISA. The experiments were performed according to the TNF- $\alpha$ ELISA kit instructions. The absorbance value was measured at $425 \mathrm{~nm}$, and the TNF- $\alpha$ levels in the serum of each group of rats were measured using a standard curve. 
Hematoxylin and eosin $(H \& E)$ staining of the liver. For histological analysis, the $10 \%$ buffered formalin fixed liver tissues was embedded in paraffin wax, sectioned $(6 \mu \mathrm{m})$ and stained with H\&E.

MicroRNA gene chip screening: MicroRNA microarray analysis. RNA was isolated from the liver in three groups rats using TRIzol, microRNA gene chip screening was performed in the model and APS groups, and differentially expressed microRNA was detected. The equipment for MicroRNA microarray experiments and analysis were provided by Shanghai Bohao Biotechnology Co., Ltd. (Shanghai, China). The chip used for the project was Agilent Rat miRNA (8x15 K) V19.0 chip (design ID: 46066). The miRNA molecules in the RNA of the experimental samples were fluorescently labeled using the Agilent miRNA microarray kit according to the standard operating procedure for labeling. The samples were hybridized according to the standard operating procedures provided with the Agilent miRNA chip and kit. The slices were washed in a wash tank after hybridization. The results of the chip were scanned using the Agilent Microarray Scanner. Data were read using the Feature Extraction software 10.7 and normalized using the Gene Spring Software 11.0. The used algorithm was Quantile.

Construction of luciferase reporter plasmid and analysis of luciferase activity. To examine whether miR-203a-3p regulated the expression of GRP78, a predicted target of miR-203a-3p, the dual luciferase psiCheck2 reporter plasmid (Promega Corporation, Madison, WI, USA), was used to generate the reporter plasmid harboring GRP78 3'-UTR. Briefly, a 387-bp fragment of GRP78 3'-UTR containing the putative miR-203a-3p binding site was amplified from rat genomic DNA by PCR, using Xho1 and Not 1 primers (left Xho1 primer: ctcgagCGGAGCAGAGCCATGGGCACGTCTTCAG, right Not 1 primer: gcggcegcCCTATTGCTGGATGCTTTCCAA GTCCC). The PCR product was digested with Xho1 and Not 1 , followed by insertion into the multiple cloning region located at the 3'-UTR of the synthetic Renilla luciferase gene within the psiCheck 2 plasmid. The psiCheck 2 plasmid also contained a synthetic firefly luciferase gene that serves as the transfection control (9)

The $293 \mathrm{~T}$ cells were co-transfected with the psiCheck2 vector containing GRP78 3'-UTR and miR-203a-3p mimic using Lipofectamine ${ }^{\circledR} 2000$ (Invitrogen; Thermo Fisher Scientific, Inc.), and the co-transfection with non-targeting negative control RNA was performed as control. The cells were harvested $24 \mathrm{~h}$ after transfection, and luciferase activity was measured with a dual luciferase reporter assay kit (Thermo Fisher Scientific, Inc.) on a luminometer (Lumat LB9507), as we described previously (9).

Reverse transcriptase-quantitative polymerase chain reaction $(R T-q P C R)$. miRNA levels in the liver and the underlying mRNA level of the target genes belonging to miR-203a-3p were determined by RT-qPCR. One gram out of overall RNA elicited by Trizol (Invitrogen, Shanghai, China) was transcribed reversely by M-MLV reverse transcriptase along with oligo-dT for mRNAs and a particular stem-loop primer as GTCGTATCCAGTGCAGGGTCCGAGGTATTC GCACTGGATACGACCTAGTGGT(5'-3'), for a miRNA.

The performance of PCR with real-time quantity was acted in a Rotor-Gene 3,000 real-time detection systems of DNA (Corbett Research Pty Ltd., Sydney, Australia) by SYBR-Green (Qiagen, Shanghai, China) using the primers as shown in Table I. All samples were analyzed in duplicate, containing no-template controls. The normalized curve method was used to determine the linked expression level of GRP78 and miRNAs, which was standardized to GAPDH and U6, respectively.

Establishment of the IR cells model. HepG2 cell lines were preserved in Typical culture preservation commission cell bank (Chinese Academy of Sciences, Shanghai, China). Hepatoblastoma HepG2 cells were cultured in DMEM medium (HyClone, Beijing, China) supplemented with $10 \%$ fetal bovine serum (HyClone) and maintained at $37^{\circ} \mathrm{C}$ in a humidified incubator with $5 \% \mathrm{CO}_{2} .24 \mathrm{~h}$ after plating, serum was removed and cells were stimulated with insulin (Gibco; Thermo Fisher Scientific, Inc.) at $100 \mathrm{~nm}$ for $48 \mathrm{~h}$. IR model were equally randomized to two groups: A APS-treatment group, and a model group. APS group were treated with APS $(10 \mu \mathrm{m} / \mathrm{ml})$, for $48 \mathrm{~h}$. And normal HepG2 cells were set as the control group.

HepG2 cells transfection. The HepG2 cells were cultured according to the method described above. The specific mimic or inhibitor RNA was transfected to overexpress or knock down miR-203a-3p expression. For transfection experiments, HepG2 cells were seeded at a density of $2 \times 9-10^{4}$ cells $/ \mathrm{cm}^{2}$ in serum-free DMEM, with addition of the transfection agent and RNAs. MiR-203a-3p mimics (40 nM) or inhibitor (40 nM) transfected to HepG2 cells for $48 \mathrm{~h}$, respectively. Then stimulated with insulin (100 $\mathrm{nM}$ ) for $12 \mathrm{~h}$, negative control oligo (NC) as the negative control. Stem-loop primer: Negative control oligo: 5'-UUCUCCGAACGUGUCACGUTT-3'; miR-203a-3p mimics: 5-GUGAAAUGUUUAGGACCA CUAG-3; miR-203a-3p inhibitor: 5'-CUAGUGGUCCUAAAC AUUUCAC-3'.

Cells were divided into six groups: Control group (normal HepG2 cells), IR+NC group (IR model transfected with negative control), IR+miR203a mimics group (IR model transfected with miR-203a-3p mimics), IR+miR203 inhibitor group (IR model transfected with miR-203a-3p inhibitor), IR+miR203 mimics + APS group (IR model transfected with miR-203a-3p mimics) and IR+miR203 inhibitor + APS group (IR model transfected with miR-203a-3p inhibitor). After $24 \mathrm{~h}$ transfection, the medium was changed and the HepG2 cells were incubated with fresh serum-containing medium for $48 \mathrm{~h}$ (Both of the two APS groups treated with APS $10 \mathrm{um} / \mathrm{ml}$ ). And then cells were harvested for protein analysis.

Detection of the protein expressions of GRP78, CHOP, JNK1, pJNK1 and caspase-12 using Western blot. The total protein of the liver tissue was extracted using the BCA protein extraction method. The same amount of total protein was used to add the loading buffer for denaturation for $3 \mathrm{~min}$. After SDS-PAGE electrophoresis, the proteins were electrotransferred onto the PVDF membrane. After blocking, the proteins were 
Table I. Primers for quantitative polymerase chain reaction.

\begin{tabular}{lll}
\hline Gene name & \multicolumn{1}{c}{ Forward primer (5'-3') } & \multicolumn{1}{c}{ Reverse primer (5'-3') } \\
\hline miR-203a & GTGCAGGGTCCGAGGTATT & GCCGCGTGAAATGTTTAGGACCAC \\
GRP78 & GGAGTGGGTCAATTCTGTTGTCA & CCCGGCACTAACGTCATTC \\
U6 & CTCGCTTCGGCAGCACA & AACGCTTCACGAATTTGCGT \\
GAPDH & AACGACCCCTTCATTGACCTC & CCTTGACTGTGCCGTTGAACT
\end{tabular}
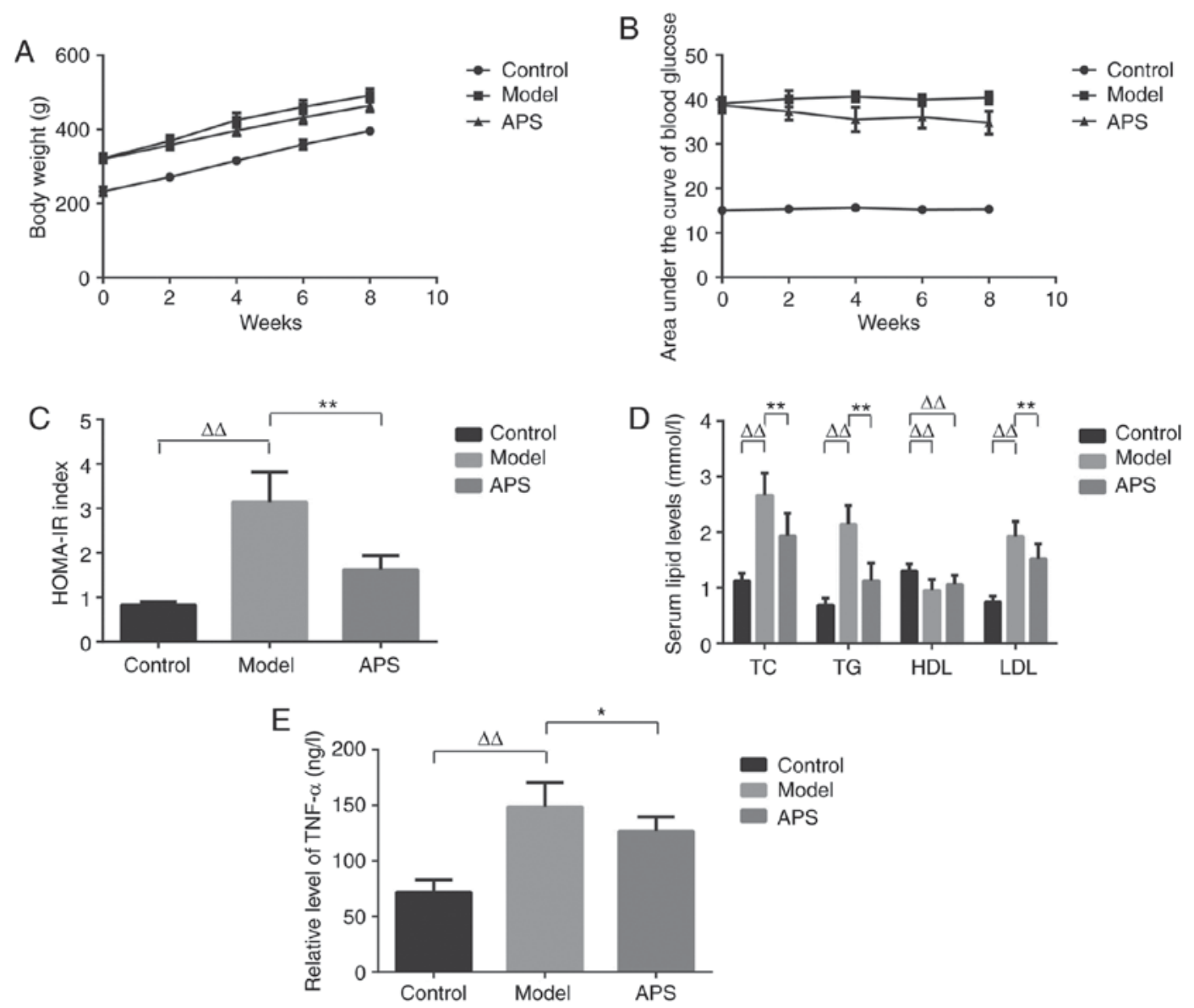

Figure 1. Effect of APS on body weight. (A) Body weight was recorded at 0, 2, 4, 6 and 8 weeks. (B) Blood glucose was recorded at 0,2, 4, 6 and 8 weeks. (C) Effect of APS on HOMA-IR index, $\mathrm{n}=10$. (D) Effect of APS on Serum lipid profile, $\mathrm{n}=10$. (E) Effect of APS on TNF- $\alpha$ level, $\mathrm{n}=10 .{ }^{\Delta \Delta} \mathrm{P}<0.01,{ }^{* * *} \mathrm{P}<0.01$ and ${ }^{*} \mathrm{P}<0.05$ as indicated. APS, Astragalus polysaccharides.

incubated with the corresponding primary antibody (GRP78, CHOP, JNK1, pJNK1 and caspase-12, diluted 1:1,000) at $4^{\circ} \mathrm{C}$ overnight, respectively. Then, they were incubated with the secondary antibody (diluted 1:7,000) at room temperature for $2 \mathrm{~h}$. After that, ECL was performed, and images were taken using the gel imaging system. Finally, the gray scale scanning analysis was performed for the results.

Statistical analysis. miRNA expression data were compared between model and APS groups by independent-sample t-test. All data are expressed as mean \pm standard deviation and analyzed by one-way ANOVA followed by LSD when Equal Variances assumed and Dunnett's-T3 when Equal Variances not assumed. Differences between groups were compared by Fisher's least significant difference (LSD). All statistical analyses were performed by using SAS 9.0 (SAS Institute, Inc., Cary, NC, USA). Two-tailed $\mathrm{P}<0.05$ showed statistical significance. Statistical comparisons were performed on GraphPad Prism 6.02.

\section{Results}

Effects of APS on body weight $(B W)$, area under the curve (AUC) of plasma blood glucose, HOMA-IR index, serum lipid levels and TNF- $\alpha$ level. The mean BW in GK rats was significantly increased as compared with that in Wistar rats of the matched age (Fig. 1A). BW of the rats in APS group was reduced by $5.6 \%$ as compared with that in model group. Area under the curve (AUC) of PBG in model group was elevated by $164 \%$ as compared with that in control groups (Fig. 1B). In 

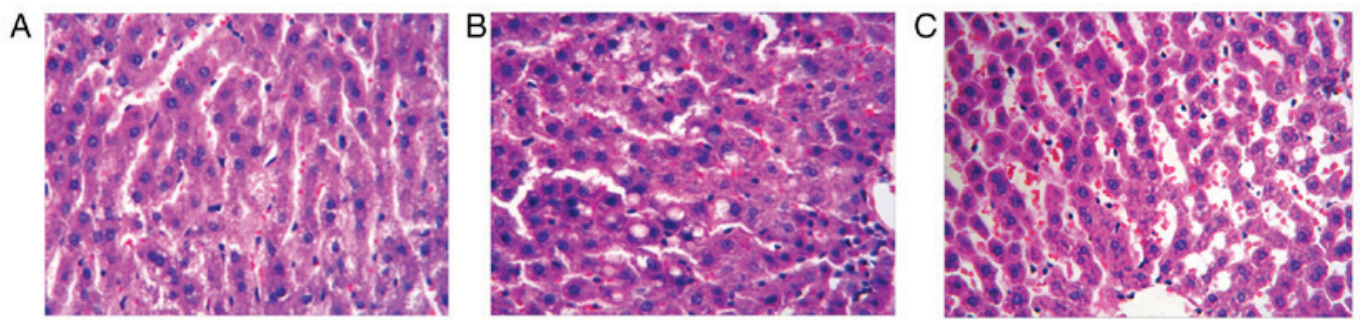

Figure 2. Effect of APS on type 2 diabetes induced changes in histopathology of rat liver. (A) Control group, (B) model group and (C) APS group Liver specimens $(n=5)$ were collected and stained with H\&E (magnification, $x 400)$.

contrast, AUC of PBG in APS group was reduced significantly by $14 \%$ as compared with that in model rats (Fig. 1B).

TC, TG and LDL levels was increased by about 2.4-, 3.1and 2.6-fold respectively in model group as compared with those in control group $(\mathrm{P}<0.01)$ (Fig. 1E). In contrast, HDL level in model group was decreased by $27 \%(\mathrm{P}<0.01)$. After APS treatment, TC, TG and LDL levels were decreased by 27,48 and $21 \%$, respectively $(\mathrm{P}<0.01)$, while HDL level showed no significant change as compared with model group $(\mathrm{P}>0.05)$. Serum level of TNF- $\alpha$ in model group were increased by about 2.1-fold respectively as compared with those in control group. After APS treatment, the level decreased by $14.7 \%$ as compared with model group $(\mathrm{P}<0.01)$ (Fig. 1F). Data are shown by mean \pm standard deviation $(n=10) .{ }^{\Delta} \mathrm{P}<0.01$, showing a significant difference as compared with control group; ${ }^{* *} \mathrm{P}<0.01$, showing a critical difference as compared with model group, employing one-way ANOVA following Tukey-Kramer multiple comparisons post hoc test.

Pathology staining of the liver. H\&E staining demonstrated that liver cells of the rats in the control group were arranged neatly, the liver lobular structure was regular and a large and round nucleus was present in the center (Fig. 2A). Moreover, the cytoplasm was uniform, and no lipid deposition was noted in the liver cells. Liver cells of the rats in the model group were swollen, and lipid droplets of various sizes were found in the cytoplasm, which were mainly larger-bubble fat droplets (Fig. 2B). Lipid droplets decreased significantly in the APS group (Fig. 2C).

Results of microRNA microarray analysis. The microRNA microarray analysis showed 12 microRNAs that had significant differences in expression in the model and APS groups. Among them, five were upregulated in the model group, including miR-103a-3p, miR-181a-5p, miR-384-3p, and so forth, and seven were downregulated, including miR-203a-3p, miR-106b-5p, and so forth. Changes of the expression of microRNA-203a-3p might be related to IR in rats (Fig. 3).

GRP78 is a target of $m i R-203 a-3 p$. To explore the mechanism by which miR-203a-3p effected on IR, we searched potential targets of miR-203a-3p using bioinformatic algorithms, such as MiRanda, Pictar and TargetScan. GRP78, a critical executioner of endoplasmic reticulum stress (ERS) signaling, was predicted as a putative target of miR-203a-3p. The identification of one miR-203a-3p-binding site was done in the 3'-UTR of GRP78 mRNA (Fig. 4A).
We determined the levels of miR-203a-3p and GRP78 mRNA. As shown in Fig. 4B and C, the miR-203a-3p levels were decreased by 0.45 -fold in model group. Interestingly, the increase in miR-203a-3p expression was most pronounced by 0.3 -fold in APS group (Fig. 4B). We further examined the expression of GRP78, one confirmed target of miR-203a-3p, along with mRNA levels by RT-qPCR. It was found that the expression of GRP78 mRNA was increased significantly in model group as compared with that in control group $(\mathrm{P}<0.05)$. On the contrary, there was no significant difference between APS and control groups (Fig. 4C).

To affirm the interrelation between miR-203a-3p and GRP78, the dual-luciferase reporter assay was implemented. Compared with the negative control RNA, the miR-203a-3p mimic significantly suppressed the activity of the luciferase reporter fused with GRP78 3'-UTR by $45 \%$ (Fig. 4D), suggesting that miR-203a-3p inhibited GRP78 expression through its 3'-UTR.

The WB result showed that GRP78 was down-regulated by miR-203a-3p, while anti-miR-203a-3p promoted GRP78 expression (Fig. 4E and F). Overall, these results revealed that miR-203a-3p could directly target and regulate the expression of GRP78.

Effect of APS on protein expressions in liver of T2DM GK rats and IR cells model. ERS is a change in the microenvironment of the endoplasmic reticulum, such as defects and disorders of protein translation, modification, turnover metabolism (10-12).

Appropriate ERS can protect cells, whereas sustained or prolonged ERS accelerates apoptosis. When ERS occurs, its classical marker GRP78 protein is highly expressed and activates three apoptotic pathways. The corresponding apoptotic mechanisms include CHOP transcriptional activation, activation of JNK signaling pathway, activation of caspase-12, and so forth (13).

We used qRT-PCR to identify the transfection effect in our preliminary experiments. All of the negative control, miR-203a-3p mimics and miR-203a-3p inhibitor had high transfection efficiency.

As demonstrated in Fig. 5, in both T2DM GK rats and IR cells model, the protein expressions of GRP78, CHOP, pJNK1, and caspase-12 were all increased significantly as compared with those in control group, respectively $(\mathrm{P}<0.01)$, while there were no significantly between APS group and control group (Fig. 5A and B). The levels of GRP78, CHOP, pJNK1, and caspase-12 proteins was decreased significantly in the clones infected with miR-203a-3p mimics in IR cells model $(\mathrm{P}<0.01)$, among which the protein expressions of those 


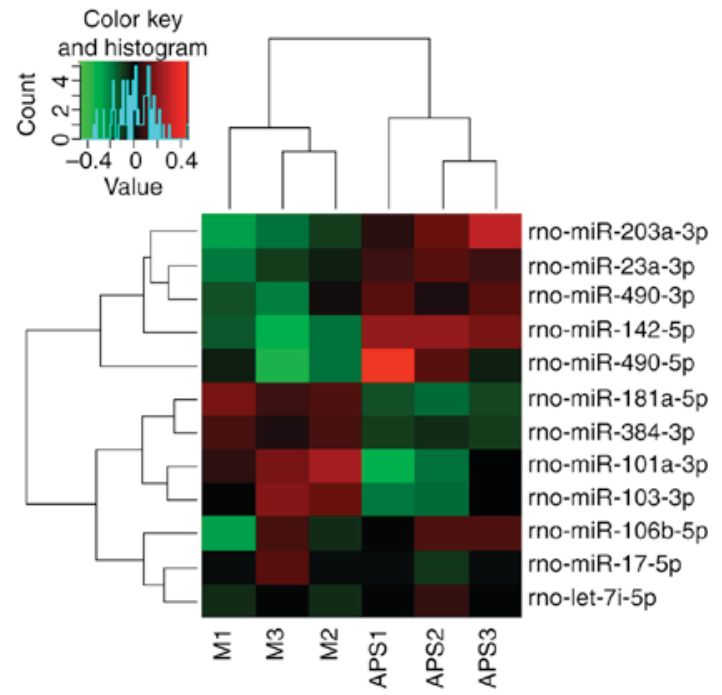

Figure 3. Heat map of differentially expressed miRNAs. Twelve differentially expressed miRNAs from the liver between the model group (n=3) and the APS group $(n=3)$. Each row represents one miRNA, and each column represents a liver tissue sample. The legend on the right indicates the miRNA represented in the corresponding row. The relative miRNA expression is depicted according to the colour scale. Red indicates upregulation, green indicates downregulation. APS, Astragalus polysaccharides.

increased significantly in the clones infected with miR-203a-3p inhibitor $(\mathrm{P}<0.01)$. After APS treated, GRP78, CHOP, pJNK1, and caspase-12 protein expressions were all decreased significantly as compared with those in model group $(\mathrm{P}<0.01)$ (Fig. 5C adn D).

\section{Discussion}

MiRNAs belong to a clan for non-coding RNAs with 20-24 nucleotides in length (14). The expression of multiple genes involving various pathways can be regulated coordinately by a singular miRNA, and therefore the gene regulation can be unraveled by discovering changed miRNA expression in complex diseases. Recent studies have found that miRNAs play an important role in the development and progression of diabetes mellitus. They are involved in blood glucose regulation, IR, islet $\beta$ cell damage and dysfunction (15). Increased number of drugs have been found to regulate miRNAs in different tissues of DM patients (16).

The Goto-Kakisaki (GK) rat is a genetic model of type non-insulin dependent diabetes mellitus without obesity. It was developed from Wistar rat with impaired glucose tolerance over successive generations. Therefore, according to the experimental routine, we chose Wistar rats as the control group. GK rats sexual maturity in 12 weeks, abnormal blood glucose usually occurs in 14-16 weeks of age, so the choice of 10 weeks old GK rats pre-feeding 6 weeks (16 weeks old) began experiment. Wistar rats of the same age had higher body weight than GK rats under the same feeding conditions. In order to better reflect the differences in body weight between the experimental group and the model group, Wistar rats with relatively small age and slightly lighter weight were selected as the normal control group. Because Wistar rat sexual maturity in 8-10 weeks, so 10 weeks of age in mature Wistar rats were tested, increased age after the biochemical indicators of the difference was not obvious, can be used as a stable control group. Also worth mentioning is that the HepG2 cell line was originally established in 1979 by Barbara Knowles and colleagues, and mistakenly reported as a hepatocellular carcinoma (17). Based on some histologic and biologic evidence that HepG2 originated as a hepatoblastoma and not a hepatocellular carcinoma $(18,19)$.

The current study aimed to investigate changes in miRNA expression in the liver of a obesity-associated T2DM GK rat model to assess their underlying devotion to disease development and progression and evaluate the effect of APS against T2DM, in an attempt to explore the mechanism of APS-affected miRNA expressions in the liver of T2DM GK rats.

Our findings indicate that particular alterations in hepatic miRNA expression existing in diabetic conditions reflect the coexisting adaptive procedure-elicited compensated IR. Analysis of the functional impact of individual changes on miRNA expression in the isolated liver revealed that some of them had beneficial effects on the activity of insulin sensitivity while others resulted in IR.

We propose that APS attenuates IR by upregulating the levels of particular miRNAs, such as miR-203a-3p, let-7i-5p, -miR-106b-5p and miR-23a-3p, miR-490-3p and miR-490-5p and downregulating miR-101a-3p, miR-103-3p, miR-181a-5p and miR-384-3p, etc. To the best of our knowledge, our study for the first time addressed the importance of miRNAs in T2DM GK rats through systematic investigations on APS's efficacy in regulating IR of miRNA changes. Our data demonstrate that IR induced by obesity is associated with alterations in two different groups of hepatic miRNAs, which have opposing phenotypic effects on the liver. These alterations in miRNA expression provide clear evidence that APS regulates hepatic miRNAs in T2DM GK rats. It was found in our study that the most striking change was the miR-203a-3p expression in model group rats. And we identified the target gene GRP78 through bioinformatic algorithms, dual-luciferase reporter assay and RT-PCR method. Thus, our primary research question was how myocardial miR-203a-3p played its negative or positive role in the genes related to ERS signaling signaling.

Recent studies have found that ERS-induced apoptosis is a new pathway of apoptosis different from the death receptor and mitochondrial pathways $(20,21)$. Under strong stimulation or sustained ERS, GRP78 is highly expressed and activates apoptosis induced by multiple ways (22). No or extremely low expression of GRP78 is found under the normal state. However, GRP78 is extremely sensitive to the endoplasmic reticulum environment. Many factors that can cause changes in the endoplasmic reticulum can directly or indirectly cause changes in GRP78 expression, such as high blood glucose, high blood pressure, oxidative stress, and inflammation, inducing the mass release of its proteins. Our data suggest miR-203a-3p upregulated by APS could reduce the expression of GRP78 mRNA and protein.

CHOP-mediated ERS apoptosis is closely related to the duration of stimulation. Gentle stimulation for a longer duration can lead to the reduction of $\mathrm{CHOP}$ level through degrading CHOP mRNA and CHOP protein to adapt to the environment (23). The activation of JNK is achieved by the Ire1-TRAF2-ASK1-JNK pathway, which promotes apoptosis by affecting Bcl-2. JNK is involved in obesity and IR-associated inflammation and oxidative stress, which plays 
A

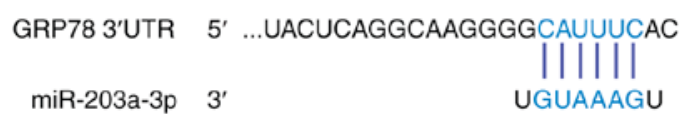

C

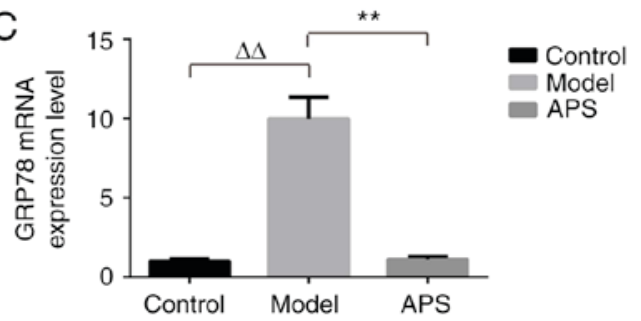

E

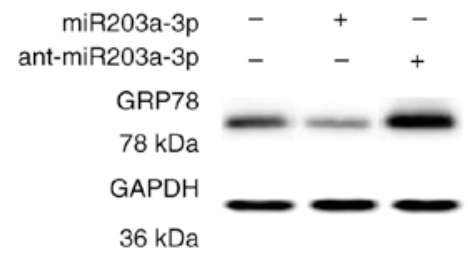

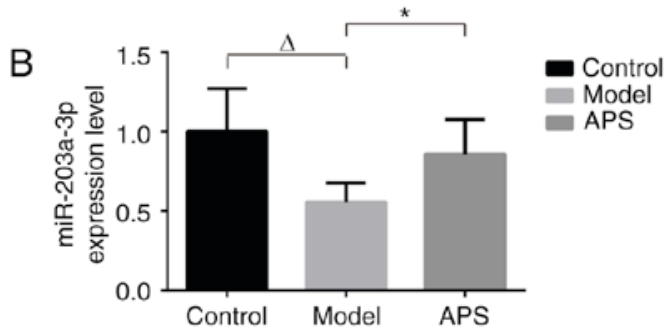
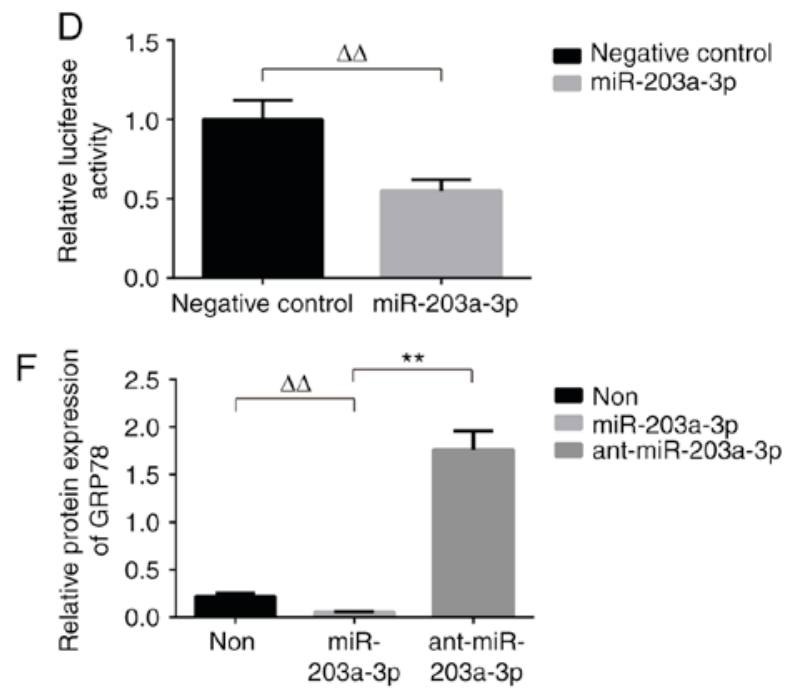

Figure 4. GRP78 is a target of miR-203a-3p. (A) The predicted binding sites of miR-203a-3p in the 3'-UTR of GRP78 mRNA. Expressions of (B) miR-203a-3p and (C) GRP78 mRNA were detected by RT-PCR analysis. (D) U6 and GAPDH were used as the internal control, respectively. $\mathrm{n}=8$. Dual-luciferase reporter assay was performed to detect the interaction between miR-203a-3p and the 3'-UTR of GRP78. $n=8$. (E and F) miR-203a-3p inhibited the protein expression of GRP78, which was detected by western blot analysis, in infected with miR-203a-3p mimics or miR-203a-3p inhibitor (anti-miR-203a-3p). GAPDH was used as the internal control. $\mathrm{n}=3 .{ }^{\Delta} \mathrm{P}<0.05,{ }^{\Delta \Delta} \mathrm{P}<0.01,{ }^{*} \mathrm{P}<0.05$ and ${ }^{* *} \mathrm{P}<0.01$ as indicated. GRP78, glucose-regulated protein 78 .

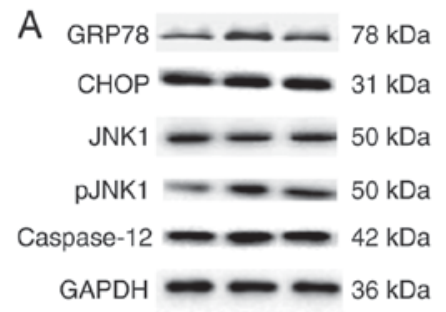

Control model APS

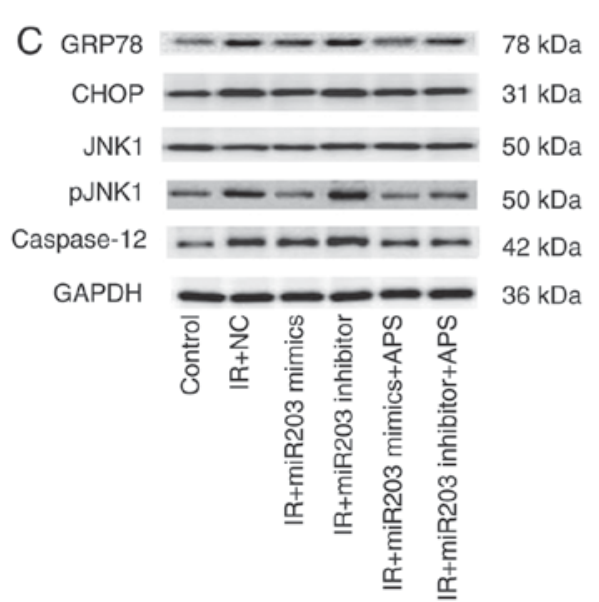

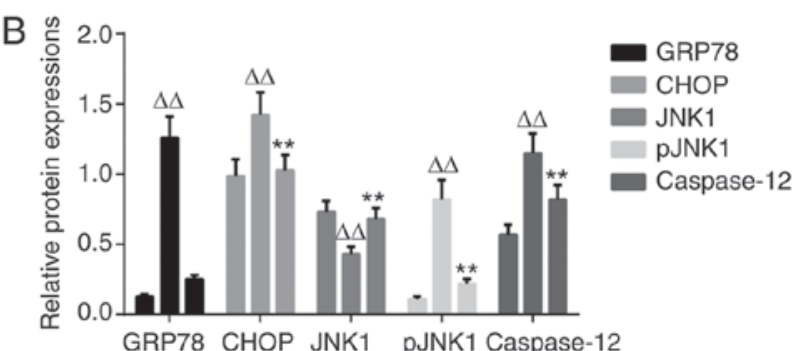

GRP78 CHOP JNK1 pJNK1 Caspase-12

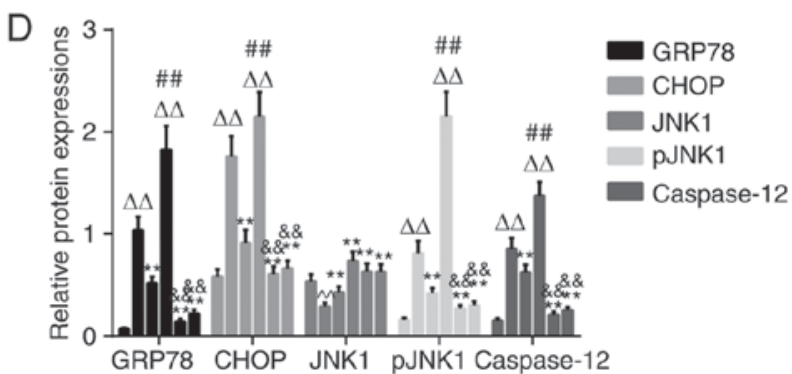

Figure 5. Effect of APS on GRP78, CHOP, pJNK1 and caspase-12 protein expressions. The protein expressions of GRP78, CHOP, pJNK1 and caspase-12 were detected by western blotting as described in the text. The figure represents one of three experiments with similar results (A and C). Data are presented as the means \pm standard deviation from three independent experiments. In (B), ${ }^{\Delta \Delta} \mathrm{P}<0.01$ vs. control group, ${ }^{* *} \mathrm{P}<0.01$, vs. model group. In (D), ${ }^{\Delta \Delta} \mathrm{P}<0.01$ vs. control, ${ }^{* *} \mathrm{P}<0.01$ vs. IR+NC, ${ }^{\#} \mathrm{P}<0.01$ vs. IR+miR203 mimics, ${ }^{\&} \mathrm{P}<0.01$ vs. IR+miR203 inhibitor. APS, Astragalus polysaccharides; GRP78, glucose-regulated protein 78; CHOP, CAAT-enhancer-binding protein homologous protein; pJNK1, phosphorylated-c-Jun N-terminal kinase 1. 
an important role in developing obesity-induced IR (24). Cysteine aspartic acid protease 12 (caspase-12) is present on the cell membrane. Studies have revealed that sustained ERS can activate pre-caspase-12, and pre-caspase-12 can be pyrolyzed into active caspase- 12 through m-calpain $\mathrm{C}$ when it encounters ERS or intracellular calcium mobilization in the endoplasmic reticulum membrane (25).

In our study, protein expressions belonging to CHOP,pJNK1, and caspase-12 in the liver were significantly increased under the condition of IR. Here, we revealed that following treatment with APS, protein expressions belonging to CHOP, pJNK1, and caspase-12 were partially restored.

Generally, our study adds new clues to the understanding about the action mechanism of APS in the treatment of IR. We propose that dysregulation and function of miR-203a-3p in the liver play an important role in IR. Overexpression of miR-203a-3p in the liver may prove to be able to attenuate IR. GRP78 is inhibited by miR-203a-3p under physiological conditions, and during the T2DM process as well. These results also imply that $\mathrm{miR}-203 \mathrm{a}-3 \mathrm{p}$ may regulate ERS signaling-related genes. Our study suggested that APS attenuated IR probably by enhancing the expression of miR-203a-3p and down-regulating the expression of GRP78 mRNA and protein and reducing the expression of CHOP, caspase-12 and phosphorylation of JNK1 protein in the liver of T2DM GK rats, which seemingly implies that the APS regulating ERS signaling might be the cause of attenuated IR.

For the first time, we discovered that APS could attenuated IR probably through up-regulating or maintaining the miR-203a-3p levels, reducing GRP78 mRNA and protein and reducing the expression of CHOP, caspase-12 and phosphorylation of JNK1 protein of the ERS signaling pathway. The research results will be benefit to better understand the effect and its mechanism in the miRNA field of APS for the treatment of diabetes.

\section{Acknowledgements}

We warmly thank Pathology Laboratory (Zhejiang Chinese Medical University, Hangzhou) for their expertise and technical help. This study was supported by grants from the National Natural Science Foundation of China (no. 81603351) and the Zhejiang Provincial Natural Science Foundation Project (no. LY16H270014).

\section{References}

1. Bacon S, Engelbrecht B, Schmid J, Pfeiffer S, Gallagher R, McCarthy A, Burke M, Concannon C, Prehn JH and Byrne MM: MicroRNA-224 is readily detectable in urine of individuals with diabetes mellitus and is a potential indicator of beta-cell demise. Genes (Basel) 6: 399-416, 2015.

2. Willeit P, Skroblin P, Kiechl S, Fernández-Hernando C and Mayr M Liver microRNAs: Potential mediators and biomarkers for metabolic and cardiovascular disease? Eur Heart J 37: 3260-3266, 2016.

3. Rawal S, Munasinghe PE, Shindikar A, Paulin J, Cameron V, Manning P, Williams MJ, Jones GT, Bunton R, Galvin I and Katare R: Down-regulation of proangiogenic microRNA-126 and microRNA-132 are early modulators of diabetic cardiac microangiopathy. Cardiovasc Res 113: 90-101, 2017.

4. Chen W, Xia YP, Chen WJ, Yu MH, Li YM and Ye HY: Improvement of myocardial glycolipid metabolic disorder in diabetic hamster with Astragalus polysaccharidess treatment Mol Biol Rep 39: 7609-7615, 2012.
5. Li GQ, Kam A, Wong KH, Zhou X, Omar EA, Alqahtani A, Li KM, Razmovski-Naumovski V and Chan K: Herbal medicines for the management of diabetes. Adv Exp Med Biol 771: 396-413, 2012.

6. Park J and Jang HJ: Anti-diabetic effects of natural products an overview of therapeutic strategies. Mol Cell Toxicol 13: pp1-20, 2017.

7. Wagner $\mathrm{H}$ and Ulrich-Merzenich G: Towards a contemporary and evidence-based development of TCM. Evid Ration Based Res Chin Drugs: pp489-516, 2012.

8. Matthews DR, Hosker JP, Rudenski AS, Naylor BA, Treacher DF and Turner RC: Homeostasis model assessment: Insulin resistance and beta-cell function from fasting plasma glucose and insulin concentrations in man. Diabetologia 28: 412-419, 1985.

9. Fang J, Song XW, Tian J, Chen HY, Li DF, Wang JF, Ren AJ, Yuan WJ and Lin L: Overexpression of microRNA-378 attenuates ischemia-induced apoptosis by inhibiting caspase-3 expression in cardiac myocytes. Apoptosis 17: 410-423, 2012.

10. Yorimitsu T and Klionsky DJ: Autophagy: Molecular machinery for self-eating. Cell Death Differ 12 (Suppl 2): S1542-S1552, 2005.

11. Shintani T and Klionsky DJ: Autophagy in health and disease: A double-edged sword. Science 306: 990-995, 2004.

12. Hamasaki M, Noda T, Baba M, Ohsumi Y: Starvation triggers the delivery of the endoplasmic reticulum to the vacuole via autophagy in yeast. Traffic 6: 56-65, 2005.

13. Yan M, Shen J, Person MD, Kuang X, Lynn WS, Atlas D and Wong PK: Endoplasmic reticulum stress and unfolded protein response in Atm-deficient thymocytes and thymic lymphoma cells are attributable to oxidative stress. Neoplasia 10: 160-167, 2008.

14. Galimov A, Hartung A, Trepp R, Mader A, Flück M, Linke A, Blüher M, Christ E and Krützfeldt J: Growth hormone replacement therapy regulates microRNA-29a and targets involved in insulin resistance. J Mol Med (Berl) 93: 1369-1379, 2015.

15. Pogribny IP, Starlard-Davenport A, Tryndyak VP, Han T, Ross SA, Rusyn I and Beland FA: Difference in expression of hepatic microRNAs miR-29c, miR-34a, miR-155, and miR-200b is associated with strain-specific susceptibility to dietary nonalcoholic steatohepatitis in mice. Lab Invest 90: 1437-1446, 2010.

16. Matsui M and Corey DR: Non-coding RNAs as drug targets. Nat Rev Drug Discov 16: 167-179, 2017.

17. Aden DP, Fogel A, Plotkin S, Damjanov I and Knowles BB: Controlled synthesis of HbsAg in a differentiated human liver carcinoma-derived cell line. Nature 282: 615-616, 1979.

18. Adesina AM, López-Terrada D, Wong KK, Gunaratne P, Nguyen Y, Pulliam J, Margolin J and Finegold MJ: Gene expression profiling reveals signatures characterizing histologic subtypes of hepatoblastoma and global deregulation in cell growth and survival pathways. Hum Pathol 40: 843-853, 2009.

19. López-Terrada D, Cheung SW, Finegold MJ and Knowles BB: Hep G2 is a hepatoblastoma-derived cell line. Hum Pathol 40: 1512-1515, 2009.

20. Paschen W and Mengesdorf T: Cellular abnormalities linked to endoplasmic reticulum dysfunction in cerebrovascular disease-therapeutic potential. Pharmacol Ther 108: 362-375, 2005.

21. Szegezdi E, Logue SE, Gorman AM and Samali A: Mediators of endoplasmic reticulum stress-induced apoptosis. EMBO Rep 7: 880-885, 2006.

22. Bertolotti A, Zhang Y, Hendershot LM, Harding HP and Ron D: Dynamic interaction of BiP and ER stress transducers in the unfolded-protein response. Nat Cell Biol 2: 326-332, 2000.

23. Oyadomari S and Mori M: Roles of CHOP/GADD153 in endoplasmic reticulum stress. Cell Death Differ 11: 381-389, 2004.

24. Ozcan U, Yilmaz E, Ozcan L, Furuhashi M, Vaillancourt E, Smith RO, Görgün CZ and Hotamisligil GS: Chemical chaperones reduce ER stress and restore glucose homeostasis in a mouse model of type 2 diabetes. Science 313: 1137-1140, 2006.

25. Liu Y, Wang J, Qi SY, Ru LS, Ding C, Wang HJ, Zhao JS, Li JJ, Li AY and Wang DM: Reduced endoplasmic reticulum stress might alter the course of heart failure via caspase-12 and JNK pathways. Can J Cardiol 30: 368-375, 2014.

This work is licensed under a Creative Commons Attribution-NonCommercial-NoDerivatives 4.0 International (CC BY-NC-ND 4.0) License. 